\title{
Epicyclic Gear Train Parametric Design Based on the Multi-objective Fuzzy Optimization Method
}

\author{
Nana Zhang ${ }^{1, a^{*}}$, Yan $\mathrm{CaO}^{2, \mathrm{~b}}$, Jiang $\mathrm{Du}^{3, \mathrm{c}}$ and Qiang $\mathrm{Li}^{4, \mathrm{~d}}$ \\ ${ }^{1}$ The Institute of Mechanical and Electrical Engineer, xi'an Technological University, Xi'an 7100321. \\ a1376194956@qq.com, bjantonyz@163.com, ‘Simen_du@sina.com
}

Keywords: Multi-Objective fuzzy optimum design; Epicyclic gear train; Pro/E parametric design

\begin{abstract}
The research can achieve multi-objective fuzzy and multi constraint optimum design of epicyclic gear train which has large transmission ratio, high power, high efficiency and simple structure, compact epicyclic gear train with multi-objective fuzzy optimum method. Based on analyzing of structure types and characteristics of epicyclic gear train, the mathematical model of fuzzy optimal design for the target with little volume and high efficiency is proposed, which can realize the non-fuzzy processing of fuzzy factors. Based on the optimization results, the parametric design process of this kind of compound gear train is realized with Pro/E, and the normal design process of this type gear is defined.
\end{abstract}

\section{Introduction}

Epicyclic gear train as a kind of compound gear train has large transmission ratio, compact structure and large transmission power, high efficiency advantages, but its design process is complex and non-standard, the feasible design scheme can be according to the single target with the no parametric method. The optimal solution is difficult to build. Recently, with the application of fuzzy technology in all fields is increasing, optimization design of gear train is a new trends. But in the fuzzy optimization design of planetary gear train, one objective fuzzy optimization design is carried out mostly with the minimum volume object, the other factors are not considered. Based on the detailed analysis and research of the basic design process of epicyclic gear train, the multi-objective and multi constraint optimization design method is realized with the advanced fuzzy technology. Based on the design result, the corresponding parametric part and assembling model are created with Pro/E, and the new gear train can be created by repaired the parametric values through the secondary development. So the development cycle can be greatly shorted.[1]

\section{The Classification and Scope of Epicyclic Gear Train}

According to the basic mechanism structure, the epicyclic gear trains can be divided into four types such as $2 \mathrm{~K}-\mathrm{H}[\mathrm{A}], 2 \mathrm{~K}-\mathrm{H}[\mathrm{B}], 2 \mathrm{~K}-\mathrm{H}[\mathrm{D}]$ and $2 \mathrm{~K}-\mathrm{H}[\mathrm{E}]$, the $2 \mathrm{~K}-\mathrm{H}[\mathrm{A}]$ epicyclic gear train is the most commonly and be studied in this paper, The structure diagram shown in fig. 1. Based on different power flow type epicyclic gear train can be divided into shunt and Reflux type. 


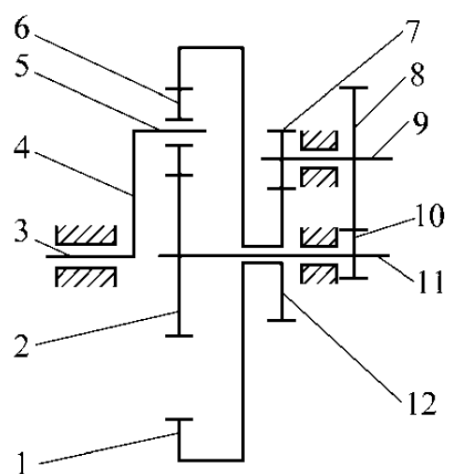

(a) Shunt type

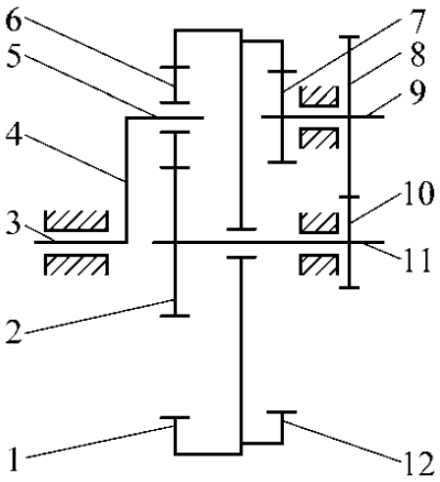

(b) Reflux type

Figure 1. The structure diagram of 2K-H[A] epicyclic gear

\section{Research and Application of Multi-objective Fuzzy Optimization System}

Mathematical Model of Multi-objective Fuzzy Optimal Design. According to fuzzy optimization theory, the small volume, high efficiency is targeted by the establishment of epicyclic gear train. Multi objective fuzzy optimal design mathematical model is established to satisfy the constraint conditions.

The Optimal Solution Model of the Volume and Efficiency Function Constraint. Each type of epicyclic gear train has its own optimal solution model of volume, efficiency function constraint, Now the process of optimal solution model establishment with the volume and efficiency function constraint is built through $2 \mathrm{~K}-\mathrm{H}[\mathrm{A}]$ Reflux type epicyclic gear train as an example to illustrate.

(1) Design variables

Design variable function

$$
X=\left(x_{1}, x_{2} \cdots x_{12}\right)^{T}=\left(z_{a}, z_{g}, z_{b}, m_{a}, \varphi_{d a}, z_{1}, z_{2}, m_{12}, z_{3}, z_{4}, m_{34}, \varphi_{d 1}\right)^{T}
$$

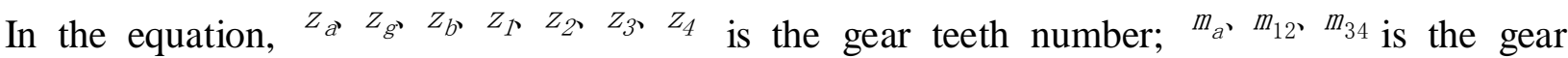
modulus; $\varphi_{d a} \varphi_{d 1}$ is the tooth width coefficient.

(2) Objective function

1) General objective function expression

$\min F(x)=(V(X), \boldsymbol{\eta}(X))^{\prime}$

In the equation, $V(x)$ is Volume function; $\eta(x)$ is Efficiency function.

2) Volume function expression

$$
V=\frac{\pi}{4} m_{\grave{l}}^{3} \varphi_{d a} Z_{a} z_{b}^{2}+\frac{\pi}{4} m_{3}^{3} \varphi_{d 1} Z_{3} Z_{4}^{2}+\frac{\pi}{4} m_{1}^{3} \varphi_{d 1} z_{1}\left(z_{1}^{2}+z_{2}^{2}\right)
$$

3) Efficiency function expression

$$
\eta=-\left[K _ { a H } \left(\eta_{a} \beta v_{a}+K_{b H}\left(\eta_{b} \beta v_{b}\right]^{\beta}\right.\right.
$$


In the equation,

$$
\begin{aligned}
& r_{a}=\operatorname{sgn} \frac{i_{a b}}{i_{a b}-i_{a b}^{H}} \\
& r_{b}=\operatorname{sgn} \frac{i_{a b}^{H}}{i_{a b}^{H}-i_{a b}} \\
& K_{a H}=\frac{i_{a b}\left(1-i_{a b}^{H}\right)}{\left[i_{a b}^{H} h^{H}{ }^{\phi}-1\right]\left(i_{a b}-i_{a b}^{H}\right)} \\
& K_{b H}=\frac{i_{a b}\left(i_{a b}^{H}-1\right)\left(h^{H}\right)^{\phi}}{\left[i_{a b}^{H} h{ }^{H} \phi-1\right]\left(i_{a b}-i_{a b}^{H}\right)}
\end{aligned}
$$

When $i_{a b}^{i^{H}}<0,0<i_{a b}^{H}<\eta^{H}$ and ${ }^{i_{a b}^{H}}>1 / \eta^{H}$

$\left.\boldsymbol{x}=\operatorname{sgn} \boldsymbol{\beta}_{b} i_{a b}^{H}\left(1-i_{a b}\right)\left(i_{a b}-i_{a b}^{H}\right)\left(i_{a b}^{H}-1\right)\right]$

When $\eta^{H}<i_{a b}^{H}<1 / \eta^{H}$

$\left.\boldsymbol{\alpha}=\operatorname{sgn}_{b}\left(i_{a b}-i_{a b}^{H}\right)\left(i_{a b}^{H}-1\right)\right\rfloor$

In the equation $\beta$ is the master slave coefficient of the tie bar $\mathrm{H}$, When the tied bar $\mathrm{H}$ is input component: $\beta=+1$; When the tied bar $\mathrm{H}$ is output component: $\beta=-1 ; \eta_{b}, \eta_{a}$ is the transfer process efficiency between the sun gear $\mathrm{a}$ 、 the sun gear $\mathrm{b}$ and the output (for the tie bar $\mathrm{H}$ is input) or input parts (For the tie bar $\mathrm{H}$ is output). $\eta^{H}$ is the conversion efficiency of the basis mechanism for differential gear train.

(3)Determination of optimal solution

The optimal solution equation

$$
\mu_{D}(X)=\max \mu_{D}(X)=\max \left[\mu_{V}(X) \wedge \mu_{n}(X)\right]
$$

The solution model is as follows:

Solution out, $\lambda, X$

The goal: $\max \lambda=\mu_{D}(X)$

Constraint as $C_{j}(X) \subset G_{j} \mathbf{j}=0,1,2, \ldots *$ (Above normal function)

$C_{j}(X) \subset G_{j}$

$\mathrm{j}=0,1,2, \ldots *$ (Above normal function)

$U_{V}(X) \geq \lambda$

(Volume fuzzy objective function)

$U_{n}(x) \geq \lambda$

(Efficiency fuzzy objective constraint)

$0 \leq \lambda \leq 1 \quad$ (Membership degree constraint)

(4)Non fuzzy processing of fuzzy constraint variables

Based on the distribution of the membership function and the comprehensive evaluation standards, Fuzzy constraint variables are transformed into non fuzzy constrained variables by fuzzy principle, and the conversion equation of specific principle in this paper does not go into detail.

The Optimal Design Example of Fuzzy Multi-objective Epicyclic Gear Train. The transmission ratio $i_{a b}^{0}=40$ known, input the speed of $960 \mathrm{r} / \mathrm{min}$ and the power of $30 \mathrm{~kW}$, the tie bar is as Follower, $\eta=0.6$ is the lowest efficiency. According to the design requirements of involute spur gear, such as precision grade 7, 45 steel materials, the number of planetary gear is $=3$.the epicyclic gear train is designed. The $2 \mathrm{~K}-\mathrm{H}[\mathrm{A}]$ gear train can be design to obtain the structural parameters by the multi-objective fuzzy optimization for small volume, high efficiency. The results design results of the gear type and related documents are the same. The carrying capacity of indicators is reflected by the smallest diameter of $\mathrm{d}_{1}, \mathrm{~d}_{2}, \mathrm{~d}_{3}, \mathrm{~d}_{4}$ of axis I, II, III, IV . Equation is: 


$$
d=17.2\left(\frac{M}{T p}\right)^{\frac{1}{3}}
$$

In the equation, $\mathrm{M}$ is the torque transmitted on the shaft. ${ }^{{ }_{p}}$ is a torsional shear stress on the shaft. The calculation of design results are shown in table 1 , Table 1 units of $\mathrm{m}^{3}$ are $V_{\min }$.

Table 1 Data results for design calculations

\begin{tabular}{ccccccccccccccccccccc}
\hline $\begin{array}{c}\text { Design } \\
\text { method }\end{array}$ & \cline { 2 - 3 } & $z_{a}$ & $z_{g}$ & $z_{b}$ & $z_{1}$ & $z_{2}$ & $z_{3}$ & $z_{4}$ & $\phi_{d a}$ & $\phi_{d 1}$ & $m_{a}$ & $m_{12}$ & $m_{34}$ & $d_{1}$ & $d_{2}$ & $d_{3}$ & $d_{4}$ & $i_{a H}$ & $\eta$ & $V_{\text {min }}$ \\
\hline $\begin{array}{c}\text { Multi } \\
\text { objetive } \\
\text { fuzzy } \\
\text { optimization }\end{array}$ & 18 & 54 & 126 & 21 & 44 & 17 & 71 & 0.75 & 0.755 & 8 & 10 & 12 & 101 & 34 & 58 & 65 & 39.99 & 0.67 & 0.204 \\
\hline
\end{tabular}

\section{Parametric System of Epicyclic Gear Train}

Parameterized Program Design of Epicyclic Gear Train. After the creation of the parts model is complete,the menu "tools / programs" option is selected, and from the "menu manager" panel , and then select "edit design" to open the program edit window, then the design parameters are defined and prompted to add to the INPUT... INPUT END, the relations add to RELATIONS. Program is process recording for the operation and this record is to save in the form of the program. In the range provided by the Program, the design of the parts is changed by modifying the parameters of the Program.[9]

Achieve the Parameters of Epicyclic Gear Train. 1) Complete assembly of the gear train of components in the Pro/ENGINEER environment, then appropriate program code and control components of assembly parameters are added to the program list generated by Pro/PROGRAM module to modify the size of the parts and replace parts, etc. Open the sample component model, use the regeneration command, call the program run, display operation menu; input: the model will be update with the input parameters of the system, as shown in Fig. 2.

2) Read the file: the parameter values are read in the file. Parameter data that need to be changed is written with notepad or writing board provided by Windows, and File extension must be DAT or TXT file. According to the prompt box of the reading file, enter the file name that read data, the new model is recycled, as shown in Fig. 3.

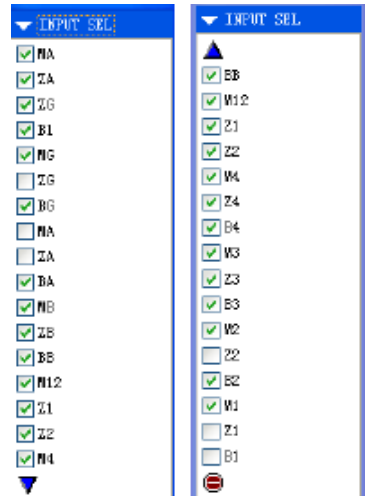

Figure 2. Display gear parameter modification

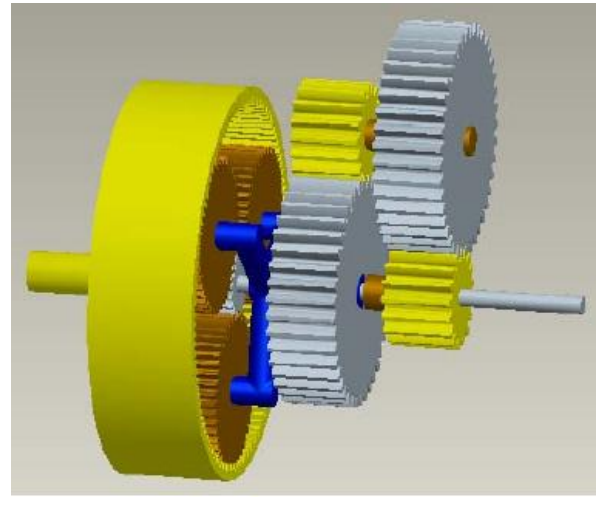

Figure 3. The new modification parameters of epicyclic gear train 


\section{Conclusions}

The parametric design method of gear train can obtain a more ideal optimization effect. The parametric design and assembly of parametric CAD system for epicyclic gear train can greatly reduce the designers' labor intensity and time, and provide a more convenient and quick way for engineering application.

\section{Acknowledgements}

The paper is supported by Key Problem Tackling Project of Shaanxi Scientific and Technological Office (2016GY-024)

\section{References}

[1] C.G. Lu and Q.H. Duan: 2K-H Power Flow, Efficiency and Self-lock for 2K-H Gear Train [J]. Mechanical design and research, Vol. 43 (2007) No.4, p. 05. (In Chinese)

[2] X. Zhou: Criterion to Judge the Power-flow Type of the Closed Epicyclic Gear Train [C]. Proceedings of international conference on mechanical transmissions. Beijing: China Machine Press: Vol. 40 (2011) No.8, p. 11.(In Chinese)

[3] Y. Yu and B. Yu: Analysis and Design of Gear Train [M]. Publishing of Harbin Engineering University, 2015.6.

[4] Giacomo $M$ and Ettore P: Theoretical and Experimental Efficiency Analysis of Multi-Degrees-of-Freedom Epicyclic Gear Trains [J].Multi-body System Dynamics.(2009) NO.9.

[5] Z. Y. Li and G. D. Chen: 3D Parametric Modeling Technology of Assembly Parts based on Pro/Engineer Platform [J]. Journal of engineering design. Vol. 40 (2013) No.4, p. 05.(In Chinese)

[6] D. B. Zhao: Parametric Design of H Closed Epicyclic Gear Train by Computer Aided Design [MS]. Jiamusi University 2007 (In Chinese)

[7] Y. B. Wang: General Numerical Method of Transmission Ratio of Epicyclic Gear Train [J]. Mechanical industry transmission. Vol. 38 (2013) No.8, p. 11. (In Chinese)

[8] Z. H Xie and X. Xun: Conversion and Transmission Ratio Calculation Method of Epicyclic Gear Train [J]. Hebei agricultural machinery. Vol. 45 (2016) No.8, p. 46. (In Chinese)

[9] M. Liu and W.H. Wang: Realize 3D Design of Epicyclic Gear Train [J]. Mechanical industry transmission. Vol. 48 (2011) No.8, p. 04. (In Chinese)

[10] Y. Y. Si: Study on Parameter Optimization of Planetary-gear Mechanism and Parametric Model of Parts [MS]. Henan Polytechnic University.2014 (In Chinese) 